\title{
Probing the Inhomogeneous Charge Distribution on Annealed Polyelectrolyte Star Polymers in Dilute Aqueous Solutions
}

\author{
Chen Qu, ${ }^{a}, \dagger$ Yi Shi, ${ }^{b, \dagger}$ Benxin Jing, ${ }^{\mathrm{c}}$ Haifeng Gao,,${ }^{\mathrm{b}}{ }^{*}$ Yingxi Zhu, ${ }^{\mathrm{c},}$ * \\ ${ }^{a}$ Department of Chemical and Biomolecular Engineering, University of Notre Dame, Notre Dame, \\ Indiana 46556 \\ ${ }^{\mathrm{b}}$ Department of Chemistry and Biochemistry, University of Notre Dame, Notre Dame, Indiana 46556 \\ ${ }^{\mathrm{c}}$ Department of Chemical Engineering and Materials Science, Wayne State University, Detroit, Michigan \\ 48202
}

\section{Supporting Information}

\section{Synthesis and Characterization of Star-Shaped P2VP}

\subsection{Materials}

2-Vinylpyridine (2-VP) (97\%, Aldrich) was dried over calcium hydride and then distilled under reduced pressure prior to use. 2,2'-Azobis(2-methylpropionitrile) (AIBN) (98\%, Aldrich) was purified by recrystallization from ethanol. Dichloromethane was freshly distilled over calcium hydride prior to use. 5(Boc-amino)-1-pentanol (>95.0\%, Aldrich), 3-(3-dimethylaminopropyl)-1-ethyl-carbodiimide hydrochloride (EDC· $\mathrm{HCl})(99 \%$, Chem-Impex), 4-(dimethylamino)pyridine (DMAP) (99\%, Aldrich), triethylamine $\left(\mathrm{Et}_{3} \mathrm{~N}\right)(>99.0 \%$, Sigma-Aldrich), 1,4-dioxane (anhydrous, 99.8\%, Sigma-Aldrich), lauroyl peroxide (LPO) (97\%, Sigma-Aldrich), trifluoroacetic acid (TFA) (99\%, Sigma-Aldrich), acryloyl chloride (97.0\%, Aldrich) were used as received. Fluorescence probes, Alexa Fluor 488 and Oregon Green 488, were purchased from Invitrogen and used as received. (Prop-2-ynyl propanoate)yl butyl trithiocarbonate was synthesized according to a reported procedure in the literature. ${ }^{1}$

\subsection{Routes of P2VP Star Polymer Synthesis}

The steps of synthesizing star-shaped poly(2-vinylpyridine) (P2VP) with an amino-functional group, which allow subsequent fluorophore labeling at precisely targeted locations on a branched arm, are detailed as follows.

Synthesis of Boc-amino Chain Transfer Agent (CTA) (1). To a round-bottom flask were added sequentially with (prop-2-ynyl propanoate)yl butyl trithiocarbonate (1.29 g, $5.4 \mathrm{mmol}$ ), 5-(Boc-amino)-1pentanol $(1.06 \mathrm{~g}, 4.9 \mathrm{mmol})$, dichloromethane $(20 \mathrm{~mL}), \mathrm{EDC} \cdot \mathrm{HCl}(1.88 \mathrm{~g}, 9.8 \mathrm{mmol})$ and DMAP $(0.30 \mathrm{~g}$, $2.45 \mathrm{mmol})$. The reaction mixture was allowed to be stirred at room temperature overnight before washed with water $(2 \times 20 \mathrm{~mL})$ and brine $(20 \mathrm{~mL})$. The organic solution was dried overnight using anhydrous 
$\mathrm{MgSO}_{4}$ overnight before removing the solvent via rotary evaporation. The final product was purified via silica column chromatography with hexane/diethyl ether (3/1 by volume) as the spreading solvent. The yield of Boc-amino CTA was $1.8 \mathrm{~g}(80 \%)$.

Synthesis of P2VP Boc-amino Macro CTA (2). The linear P2VP Boc-amino macro-CTA was synthesized by reversible addition-fragmentation chain transfer (RAFT) polymerization using (1) as RAFT agent and AIBN as initiator. A typical procedure is as follows: as illustrated in Scheme S1, (1) (0.404 g, $0.635 \mathrm{mmol}), 2-\mathrm{VP}$ monomer (15.0 g, $0.143 \mathrm{~mol}$ ), and AIBN (23.4 mg, $0.0952 \mathrm{mmol}$ ) were added into a $25 \mathrm{~mL}$ flask. The mixture was degassed through four freeze-evacuate-thaw cycles before sealing the flask under vacuum. The polymerization was carried out in an oil bath at $70{ }^{\circ} \mathrm{C}$ for $2.5 \mathrm{~h}$. The reaction was terminated by cooling down to room temperature and exposing to air. The resulting crude product was purified by precipitation into large amount of hexane three times. The final product was dried under reduced pressure to a constant mass with yield of $7.5 \mathrm{~g}$.

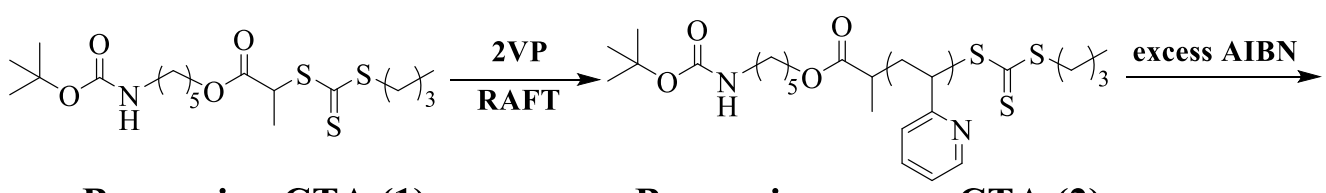

Boc-amino CTA (1)

Boc-amino macro CTA (2)

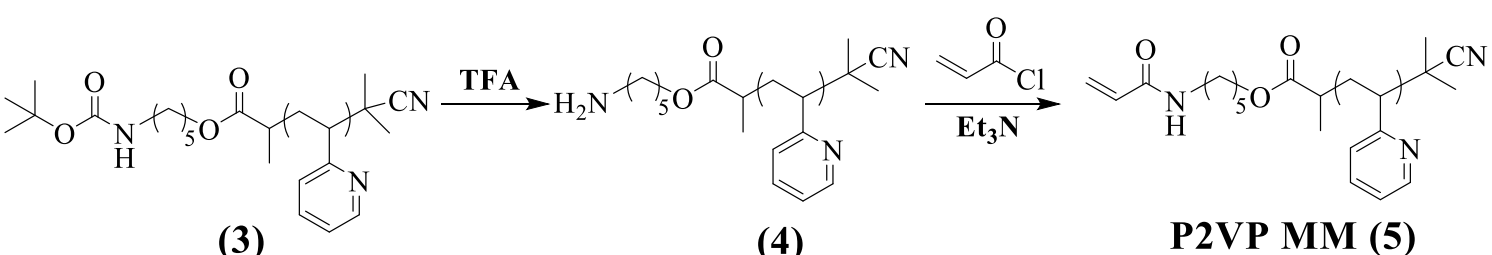

(3)

(4)

\section{P2VP MM (5)}

Scheme S1. Synthesis of P2VP macromonomer (MM) from Boc-amino macro CTA via terminal modification.

Removal of the Thiocarbonylthio Group. The thiocarbonylthio group was removed from linear P2VP according to a published protocol. ${ }^{2}$ A mixture of (2) (6.0g), AIBN (2.460 g, 20 molar equivalents), LPO (0.598 g, 2 molar equivalents) and 1,4-dioxane $(80 \mathrm{~mL})$ was placed in a dried flask. The solution was degassed through four freeze-evaluate-thaw cycles, sealed, and heated at $80^{\circ} \mathrm{C}$ for $4 \mathrm{hrs}$. The reaction was terminated by cooling down to room temperature and exposing to air. The resulting crude product was purified by precipitation into diethyl ether twice and hexane once. The final product, P2VP without RAFT agent end group (3), was dried under reduced pressure to a constant mass with yield of $4.7 \mathrm{~g}$.

Deprotection of the Tert-butoxycarbonyl (Boc) Group. A typical procedure to remove the Boc group in P2VP polymer was as follows: P2VP (1) (4.0 g), trifluoroacetic acid (TFA) (4.74 g, 1.1 molar 
equivalents of 2VP monomers in P2VP (1) and water (35 mL) were added into a flask. The mixture was vigorously stirred for $3 \mathrm{hrs}$. The solution was then slowly added to $\mathrm{Na}_{2} \mathrm{CO}_{3}$ solution to adjust to the pH to

$\sim 8$ to get crude products as deposits. The resulting crude product was subsequently purified by precipitation into hexane three times. The final product P2VP (4) was dried under reduced pressure to a constant mass with yield of $3.7 \mathrm{~g}$. The procedures for removal Boc group in star-shaped P2VP are similar to that described above.

Synthesis of P2VP MM (5). As illustrated in Scheme S1, P2VP (4) (3.3 g), triethylamine (0.833 g, 20 molar equivalents) and dichloromethane $(40 \mathrm{~mL})$ were added to a dried flask followed by adding acryloyl chloride $(0.373 \mathrm{~g}, 10$ molar equivalents $)$ dropwise at $0{ }^{\circ} \mathrm{C}$. The reaction was kept at room temperature overnight, followed by adding water to wash the mixture $(3 \times 20 \mathrm{~mL})$. The organic layer was collected and dried over magnesium sulfate. The resulting crude product was then purified by precipitation into hexane three times. The final product was dried under reduced pressure to a constant mass with yield of $2.8 \mathrm{~g}$.

Synthesis of Star-Shaped P2VP with Different Labeling Sites by RAFT Polymerization. The procedure to prepare star-shaped P2VP with Boc group at the star center was as follows: (5) (0.20 g), (1) (1.3 mg, 0.125 molar equivalents), AIBN $(0.08 \mathrm{mg}, 0.47 \mathrm{mmol})$ and 1,4-dioxane $(3 \mathrm{~mL})$ were added into a $20 \mathrm{~mL}$ flask. The mixture was degassed through four freeze-evacuate-thaw cycles before sealing the flask under vacuum. The polymerization was carried out in an oil bath at $70{ }^{\circ} \mathrm{C}$ for $8 \mathrm{hrs}$. The reaction was terminated by cooling down to room temperature and exposing to air. The resulting crude product was purified by precipitation into hexane three times. The final product was dried under reduced pressure to a constant mass with yield of $0.14 \mathrm{~g}$.

The procedure for preparing star-shaped P2VP with Boc-amino group at the terminal group of one of the arms was similar to that described above except a (2) was used as RAFT agent at a feed ration of [P2VP $\mathrm{MM}]_{0}:[\mathrm{P} 2 \mathrm{VP} \text { Boc-amino macro CTA }]_{0}:[\mathrm{AIBN}]_{0}=7: 1: 0.2$.

\section{3. Characterization}

Dimethylformamide (DMF) size exclusion chromatography (SEC) system includes a Waters 515 High-performance liquid chromatography (HPLC) pump, a Waters 2414 refractive index detector, three Polymer Standards Services (PSS) columns (GRAM) with a flow rate of $1.00 \mathrm{~mL} / \mathrm{min}$ at $55^{\circ} \mathrm{C}$. Linear poly(methyl methacrylate) (PMMA) standards were used for calibration. Nuclear magnetic resonance $(\mathbf{N M R})$ spectra were recorded on a Bruker $\mathrm{DM} \times 400$ spectrometer using $\mathrm{CDCl}_{3}$ as the solvent. UV-vis Absorption spectra were collected either on Perkin Elmer Lambda 25 spectrometer with UVWin Lab software or evolution $201 \mathrm{UV} /$ Vis Spectrometer with thermo insight software. Spectra data were obtained using spectrophotometric grade solvent at $20{ }^{\circ} \mathrm{C}$ with glass cuvette $(1 \mathrm{~mL}, 10 \mathrm{~mm}$ path 
length).

\section{Preparation and Characterization of P2VP Stars with Fluorophore Labeling}

\subsection{Fluorophore Labeling P2VP Stars.}

The center and arm terminal labeled P2VP stars were chemically attached with a fluorophore, Alexa Fluor 488 (Alexa488) or Oregon Green 488 (OG488), both from Invitrogen, to the amino endfunctionalized group according to a published protocol. ${ }^{3}$ After the reaction with the fluorophores, P2VP star polymers were purified through centrifuge filter in deionized water at $\mathrm{pH} \sim 2$ to remove excessive fluorescence molecules. Subsequently, P2VP aqueous solutions were prepared by dissolving fluorophore labeled $\mathrm{P} 2 \mathrm{VP}$ in $\mathrm{HCl}$ solution with a resulting $\mathrm{P} 2 \mathrm{VP}$ molar concentration of $\sim 10^{-9} \mathrm{M}$ to ensure a sufficiently low concentration to reach the single molecular level for fluorescence correlation spectroscopy (FCS) measurement. The solution pH, determined by an Oakon pH6 meter, was controlled by the concentration of added $\mathrm{HCl}$ to maintain the possibly lowest ionic strength upon varying $\mathrm{pH}$.

\subsection{Characterization of the Fluorophore Labeling Efficiency.}

After purifying the fluorophore labeled P2VP star, the sample was freeze-dried to completely remove solvents. The sample was then weighed and dissolve in a dilute aqueous solution with known polymer molar concentration, $c_{1}$. The concentration of fluorophore-labeled P2VP star, $c_{2}$, in the same solution was measured by FCS. As only the fluorophore-labeled polymers can be detected by FCS and unlabeled P2VP stars make no contribution to the measured profile of fluorescence intensity fluctuation, the efficiency of fluorescence labeling with P2VP stars is thus obtained by the ratio $c_{2} / c_{1}$. The results are shown below in Table S1 in Supporting Results.

\section{Experimental Characterization Methods.}

The diffusive dynamics of fluorophore-labeled P2VP star polymers in aqueous solutions was characterized by FCS at a single molecule level. ${ }^{4-6}$ The experimental setup is based on an inverted microscope (Zeiss Axio A1) equipped with an oil-immersion objective lens (Plan Apochromat 100×, NA $=1.4)$. Briefly, the tiny fluctuations, $I(t)$ in fluorescence intensity, due to the motion of fluorescent probes in and out of the laser excitation volume, with an Argon ion laser (Melles Griot, $\lambda_{e x}=488 \mathrm{~nm}$ ) were measured by two single-photon counting modules (Hamamatsu) independently in a confocal detection geometry at a sampling frequency of $100 \mathrm{kHz}$ in this work. The dimension of the excitation confocal volume was calibrated as $\bar{\omega} \sim 260 \mathrm{~nm}$ in the lateral diameter and $\mathrm{z} \sim 2 \mu \mathrm{m}$ in the vertical height by Alexa Fluor 488 in a dilute aqueous solution. The auto-correlation function, $G(\tau)$ of measured $I(t)$ as $G(\tau)=$ $\langle\delta I(t) \cdot \delta I(t+\tau)\rangle /\langle I(t)\rangle^{2}$ (Eq.1) was thereby obtained by using a multichannel FCS data acquisition 
system (ISS) via cross-correlation analysis. The diffusion coefficient, $D$ and the probe concentration in the focal volume, $[c]$ were obtained by fitting $G(\tau)$ with the Eq. 2 below assuming 3D Brownian diffusion:

$$
\mathrm{G}(\tau)=\left([c] \pi^{2} z^{2} \bar{\omega}\right)^{-1}\left(1+\frac{4 D \tau}{\bar{\omega}^{2}}\right)^{-1}\left(1+\frac{4 D \tau}{z^{2}}\right)^{-0.5}(\text { Eq. } 2)
$$

In this work, the measurements were repeated three times for each sample at a given solution $\mathrm{pH}$ to obtain the average values of $D$ and $[c]$. All the experiments were conducted at $20{ }^{\circ} \mathrm{C}$.

Photon counting histogram (PCH) analysis was employed based on the same FCS optical setup but to obtain information on the brightness of fluorescent molecules, whose principle has been detailed elsewhere. ${ }^{6-7}$ Basically, PCH analyzes the distribution of fluorescence emission within the excitation confocal volume, for instance, the histogram of fluorescence photon counts. With the fluctuation in the number of fluorophores in the excitation-detected volume, such as via Brownian motion, the distribution of fluorescence photon counts, i.e. the probability, $\mathrm{p}(\mathrm{k})$ of detecting $\mathrm{k}$ photon counts at a given time can be fitted with a "super-Poisson" function. By analyzing the distribution of fluorescence emission, the average number of fluorophores within the confocal volume and the brightness in the unit of photon counts per second per molecule (CPSM) can be obtained.

Quantum yield measurement was conducted by Horiba-Yovan Fluoromax 4 spectrometer with a slit width of $2 \mathrm{~nm}$ to confirm the observation by PCH. OG488 in aqueous solution with its known absolute quantum yield, $\emptyset_{S}$, at $\mathrm{pH}=7$ was used as the standard reference for the measured relative

quantum yield, $\emptyset_{i}=\emptyset_{S} \frac{A_{i} f_{S}}{A_{S} f_{i}}$ (Eq. 3), where $\emptyset_{i}$ is the measured absolute quantum yield of the test sample, $\mathrm{A}$ is the absorbance and $\mathrm{f}$ is the integration of fluorescence emission area over wavelength. The subscripts $i$ and $s$ denote the test sample and standard reference, respectively. In all the measurements, $A_{i}$ was controlled in a narrow range within $0.05 \sim 0.10$ as confirmed by UV-vis spectra. The concentration of all the test samples was $10^{-6} \mathrm{M}$. $\emptyset_{i}$ was calculated from the condition of $\emptyset_{S}=0.97$ and $A_{S}=0.08$ at $\mathrm{pH}=7.0$ in aqueous solution.

\section{Supporting Results}

\subsection{Characterization Results of Synthesized P2VP Star Polymers with Their Fluorophore Labeling}

(1) was synthesized by esterification between (prop-2-ynyl propanoate)yl butyl trithiocarbonate with 5-(Boc-amino)-1-pentanol, and confirmed by ${ }^{1} \mathrm{H}$ NMR and ${ }^{13} \mathrm{C}$ NMR, as shown in Figure S1. The degree of polymerization (DP) of P2VP macro CTA estimated by monomer conversion according to ${ }^{1} \mathrm{H}$ NMR spectra is 75 .

Figure S2 (A) shows the SEC profiles of linear P2VP during terminal modification from P2VP macro CTA (2) to P2VP MM (5). For all these four products, the molecular weight distribution $\left(M_{\mathrm{w}} / M_{\mathrm{n}}\right)$ was below 1.18 with a same elution volume, indicating constant molecular weight and size after each step. 
The chemical structure of these four products during terminal group modification was confirmed by ${ }^{1} \mathrm{H}$ NMR spectra as shown in Figure 2S (B).
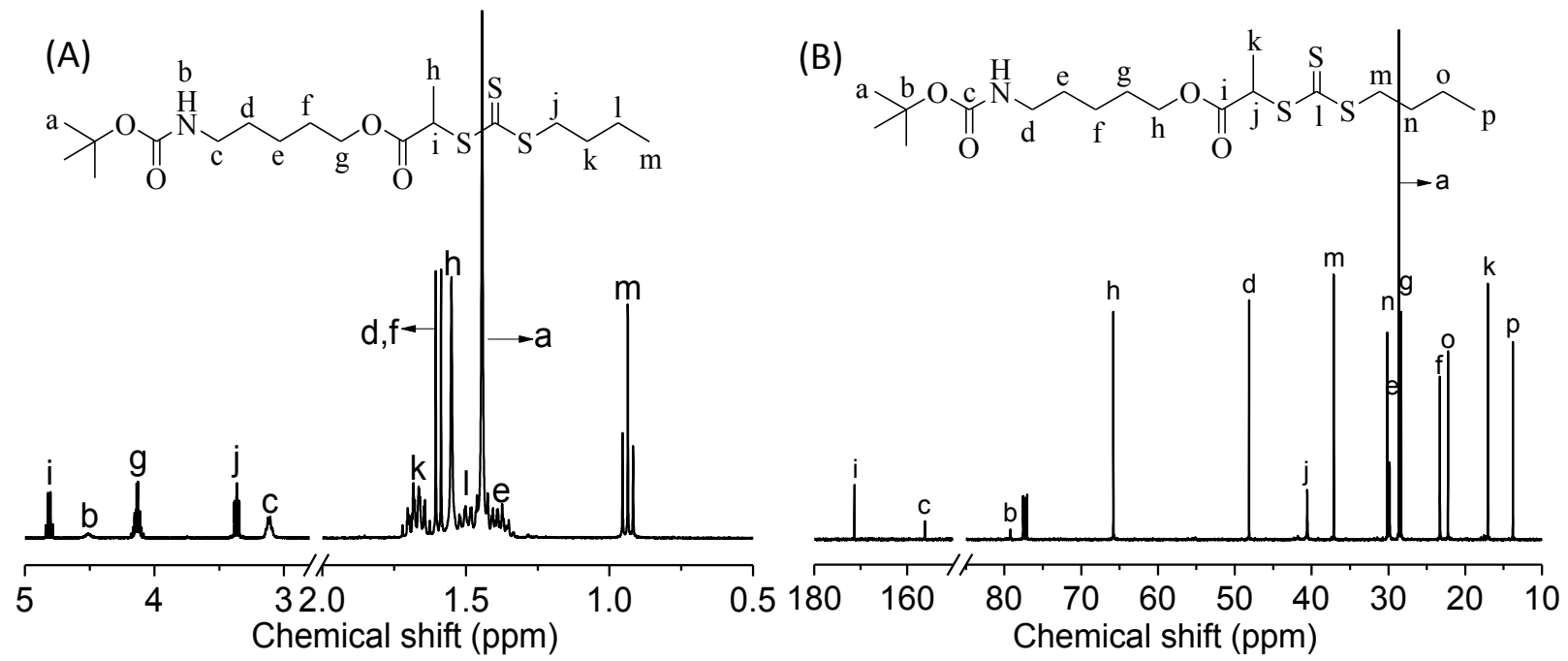

Figure S1. $(A)^{1} H$ NMR and $(B){ }^{13} C N M R$ spectra of Boc-amino CTA.
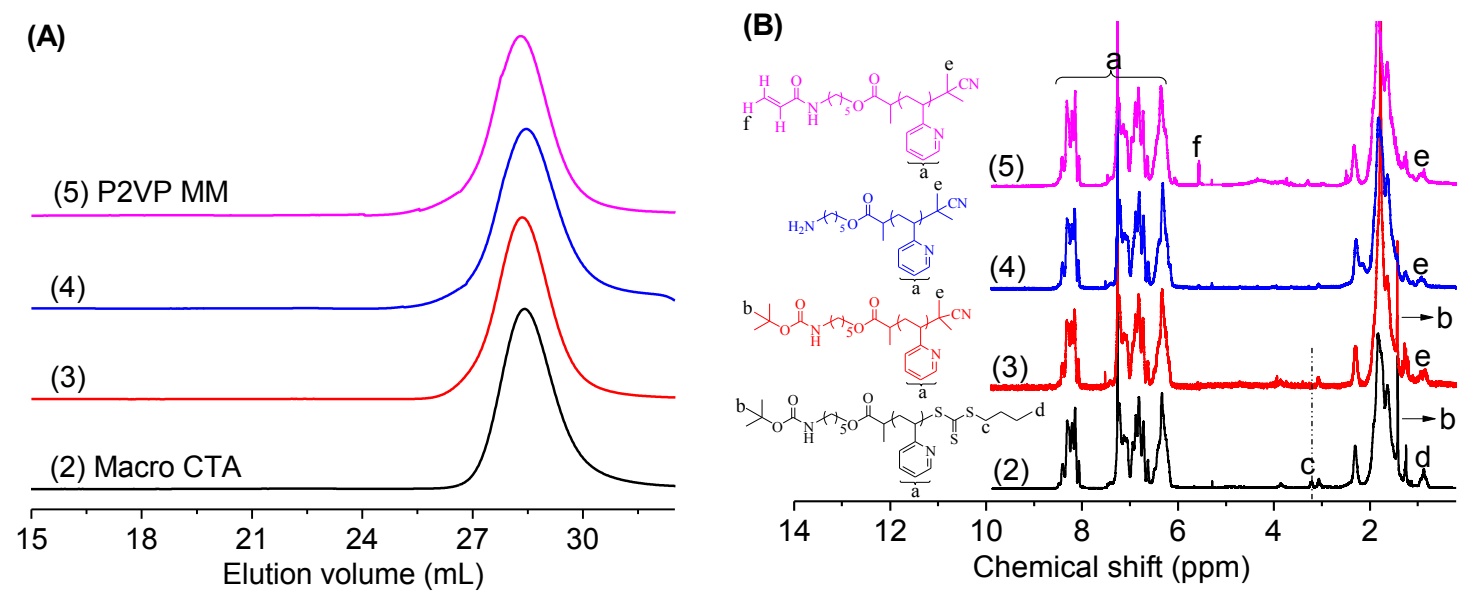

Figure S2. (A) SEC traces and (B) ${ }^{l} H$ NMR spectra of P2VP with different functional groups.

The deprotection of Boc group from the synthesized P2VP star polymers was confirmed by ${ }^{1} \mathrm{H}$ NMR spectra, as shown in Figure S3. After removing the Boc protection group, the peak at $1.42 \mathrm{ppm}$ (Boc group) completely disappears, meaning that the free amine group was released. Subsequently, Alexa488 or OG488 with activated ester group, were covalently attached to P2VP star at either center or periphery via ester-amine coupling. 


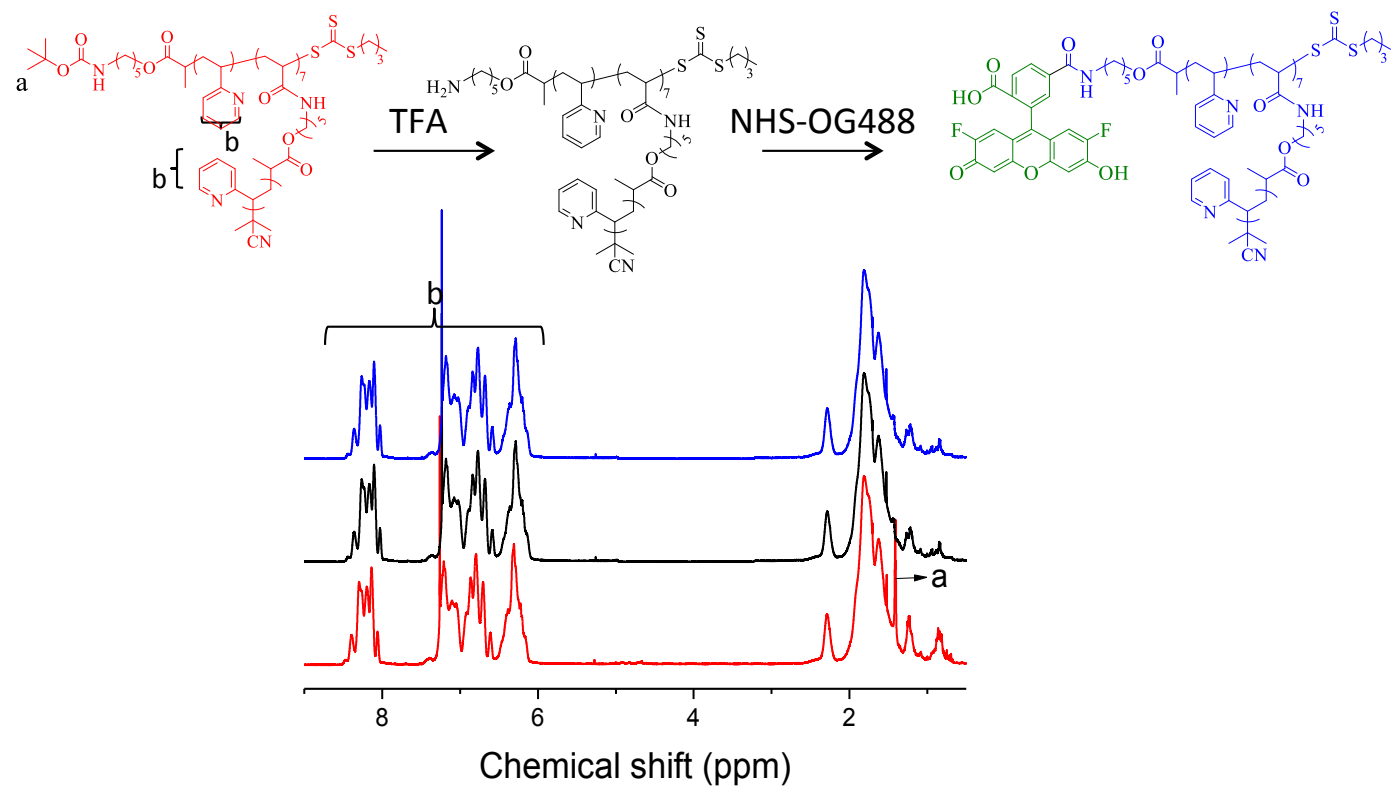

Figure S3. ${ }^{1} H$ NMR spectra of P2VP stars before (red) and after (black) Boc-deprotection with

fluorophore OG488 labeling site at one arm terminal (blue).

The reaction of activated ester of fluorophores with amine groups in P2VP stars was confirmed by UV-vis spectroscopy and FCS. It is noted ${ }^{1} \mathrm{H}$ NMR spectroscopy may not be a good tool to monitor the reaction because the chemical shifts of most protons in Alexa488 or OG488 overlap with those of pyridine groups in the polymer (Figure 1B and Figure S3). As shown in Figures S4, P2 VP star polymers after fluorophore labeling show strong absorption peaks at $500 \mathrm{~nm}$ evidently, indicating the successful attachment of fluorophores to P2VP star polymers in the center (blue) or the end of an arm chain (green). The procedure to measure the efficiency of fluorescence labeling is described in the Supporting Information Section 2.2 and the results are summarized in Table S1. It is found that for all polymer samples, the efficiency was above 0.4 , which is sufficiently high for FCS measurement to give reliable results. 

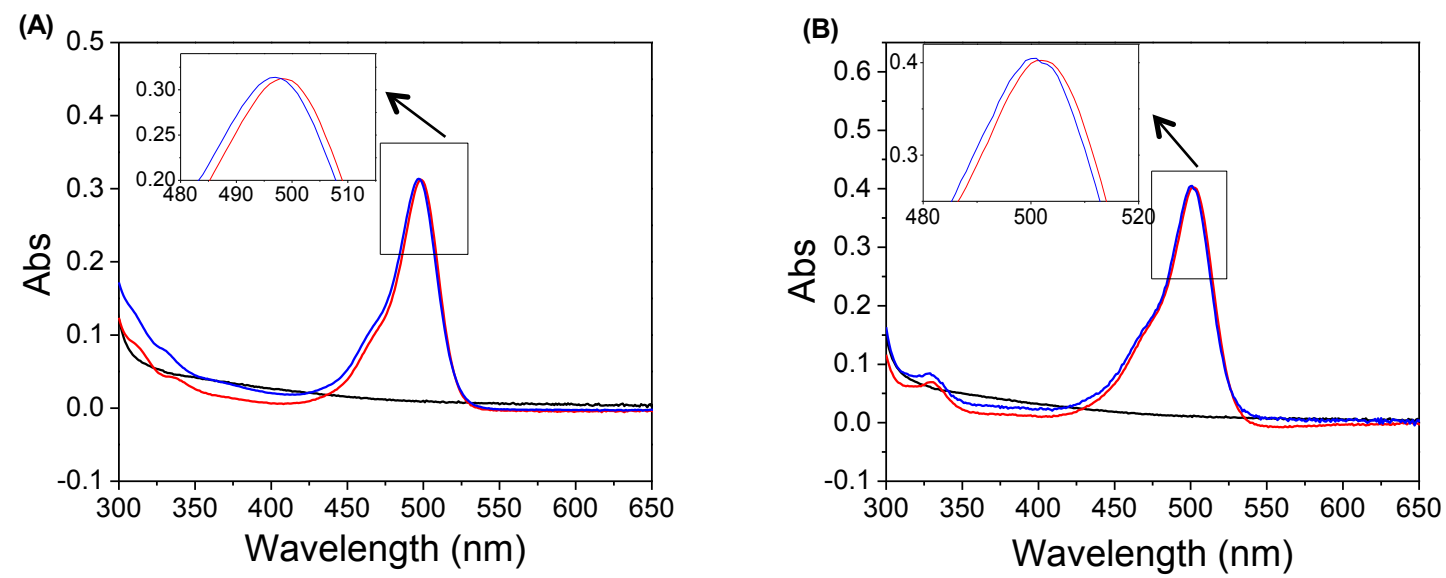

Figure S4. UV-vis absorption spectra of P2VP stars before (black) and after fluorophore labeling in the center (blue) and the end of an arm chain (red) with A) alexa488 and B) OG488. (Inset) The blow-out of the peak regions.

Table S1 Characterization of Fluorophore-labeled P2VP stars by NMR ${ }^{a}, S^{2} C^{b}$ and FCS ${ }^{c}$.

\begin{tabular}{|c|c|c|c|c|c|}
\hline \multirow{2}{*}{ Sample } & $M n^{a}$ & & $M^{a, b, *}$ & $M_{w} / M_{n}{ }^{\mathrm{b}}$ & \multicolumn{2}{|c|}{ Labeling Efficiency $^{\mathrm{c}}$} \\
\cline { 5 - 6 } & & & & Alexa488 & OG488 \\
\hline Arm-T & $8.0 \mathrm{~K}$ & $8.0 \mathrm{~K}$ & 1.18 & - & - \\
\hline Star-C & $60 \mathrm{~K}$ & $64 \mathrm{~K}$ & 1.30 & 0.43 & 0.40 \\
\hline Star-T & $62 \mathrm{~K}$ & $64 \mathrm{~K}$ & 1.28 & 0.62 & 0.65 \\
\hline
\end{tabular}

*Estimated from the feed ratio and conversion: 1) for the arm, the feed ratio of 2-VP monomer to CTA for polymerization is $150: 1$ with the productivity of $50 \%$, so the $\mathrm{N}$ is estimated to be 75 with $\mathrm{Mn}=8 \mathrm{~K}$. 2) For the P2VP stars formation from P2VP MM, the feed ratio of MM to Macro-CTA and CTA is 7:1 and $8: 1$, respectively. From SEC curve the conversion is $\sim 1$. The theoretical Mn for P2VP stars is $64 \mathrm{~K}$.

\subsection{Conformational Structure of P2VP Stars}

The conformational structures of P2VP stars in aqueous solutions of varied $\mathrm{pH}$ were described by measured hydrodynamic radius, $\mathrm{R}_{\mathrm{H}}$ as shown in Figure $2 \mathrm{~B}$. Here we semi-quantitatively compare our results with the theoretical scaling prediction. ${ }^{8-10}$ For an annealed $\mathrm{PE}$ star with $p$ arms of each polymerization degree, $\mathrm{N}\left(=76\right.$ for P2VP $\mathrm{MM}$ of $\left.\mathrm{M}_{\mathrm{n}}=8,000\right)$ and ionization degree, $\alpha$, when the total charge number, $Q=N p \alpha$, is smaller than the ratio of its radius, $R$ to Bjerrum length, $l_{B}(=0.7 \mathrm{~nm}$ in deionized water), counterions were modeled to distribute uniformly in the solution outside the star PE, 
leading to unscreened electrostatic repulsion between monomers, designated as the polyelectrolyte regime. ${ }^{8-10}$ The radius of the PE star of its monomer size, $a$ in the polyelectrolyte regime was predicted as $R \sim a N \alpha^{2 / 3}\left(l_{B} / a\right)^{1 / 3} p^{1 / 3}$ (Eq. 4). ${ }^{9}$ The star PE became more stretched with the power function of $p^{1 / 3}$ than linear PEs in salt-free aqueous solution. As increasing arm number, counterions were modeled to be mostly trapped inside the inter-arm volume and thereby neutralize the monomer charges, designated as the osmotic regime. The osmotic pressure of confined counterions together with polymer conformational entropy gave rise to a saturated length of stretched arms independent of arm number as $R \sim a N \alpha^{1 / 2}$ (Eq. 5). ${ }^{9}$ The crossover between the polyelectrolyte and osmotic stars occurred at a critical arm number, $p^{*} \sim \alpha$ ${ }^{-1 / 2}\left(l_{B} / a\right)^{-1}$ (Eq. 6). To meet the criteria of $Q<R / l_{B}$, we estimated $\alpha<0.8 \%$ based on measured $R_{H}=3.5 \mathrm{~nm}$ for swollen P2VP stars of $p=8$. Apparently, such a low $\alpha$ was not practical for P2VP at low $\mathrm{pH}(<3)$ considering $\mathrm{pKa}=6.12$ for the protonated 2-ethylpyridine monomer, and we considered P2VP stars bearing 8 arms at low pH was preferably in the osmotic regime. Future work with P2VP stars of varied arm number and length will be conducted to examine the theoretical scaling predictions.

\subsection{Confirming the PCH Results with P2VP Stars Labeled with Fluorophores at Different Locations.}

Effect of Labeling Location on the Fluorescence Characteristics of Fluorophores. To verify that the distinct brightness and derived local $\mathrm{pH}$ of P2VP stars labeled with OG488 at different locations are resulted from the local electrostatic environment in the close vicinity to P2VP segments, not the possible artifact brought by chemical linkage of the fluorophore to the P2VP star, we determined the steady-state fluorescence emission spectra of free OG488 and its conjugation with P2VP star in the center and the periphery at different $\mathrm{pH}$. As shown in Figure S5, the spectra for OG488 attached to the center and the periphery of a P2VP star at chosen solution $\mathrm{pH}$, whose corresponding local $\mathrm{pH}$ is the same as the bulk $\mathrm{pH}$ chosen for the measurement of free OG488, overlap with that of free OG488, confirming the negligible effect of labeling location on the fluorescence emission characteristics of fluorophore. 
(A)

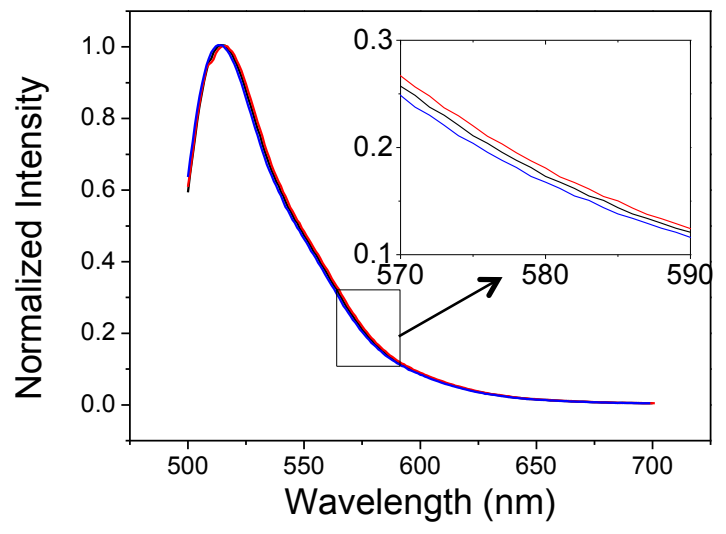

(B)

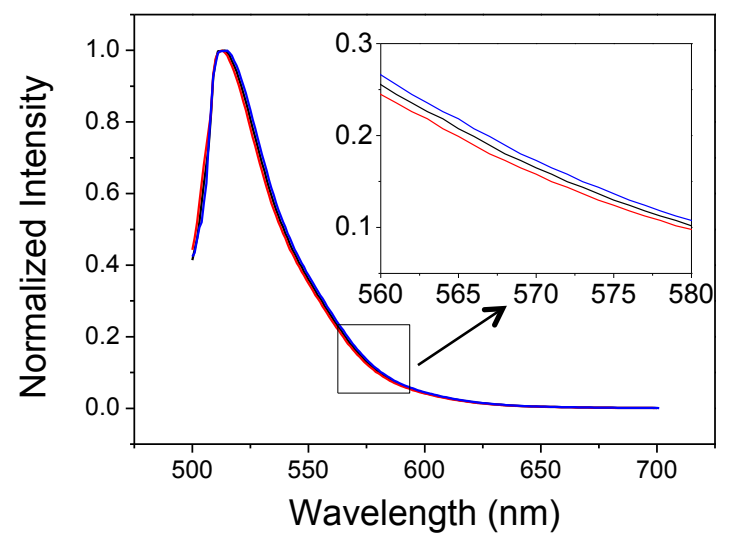

Figure S5. Normalized fluorescence emission spectra of (A) free OG488 (blue) at $p H=4.9$ and OG488 attached to the center (red) at $p H=2.4$ and periphery (black) at $p H=2.6$ of a P2VP star in aqueous solution. B) Free OG488 at $\mathrm{pH}=5.5$ and OG488 attached at center under $\mathrm{pH} 2.8$ and OG488 attached at periphery under $\mathrm{pH} 3.2$.

In this work, we also used a pH-independent fluorophore, Alexa488, to label P2VP stars at different locations. Similar results of fluorescence emission spectra were obtained with Alexa488 attached to the center and periphery of P2VP star, further confirming the negligible effect of polymer attachment on the fluorescence characteristics of fluorophores. As the fluorescence emission of Alexa488 is independent of solution $\mathrm{pH}$, the measured brightness of Alexa488-labeled P2VP stars is constant against solution $\mathrm{pH}$, in sharp contrast to that of OG488-labeled ones as compared in Figure S6.

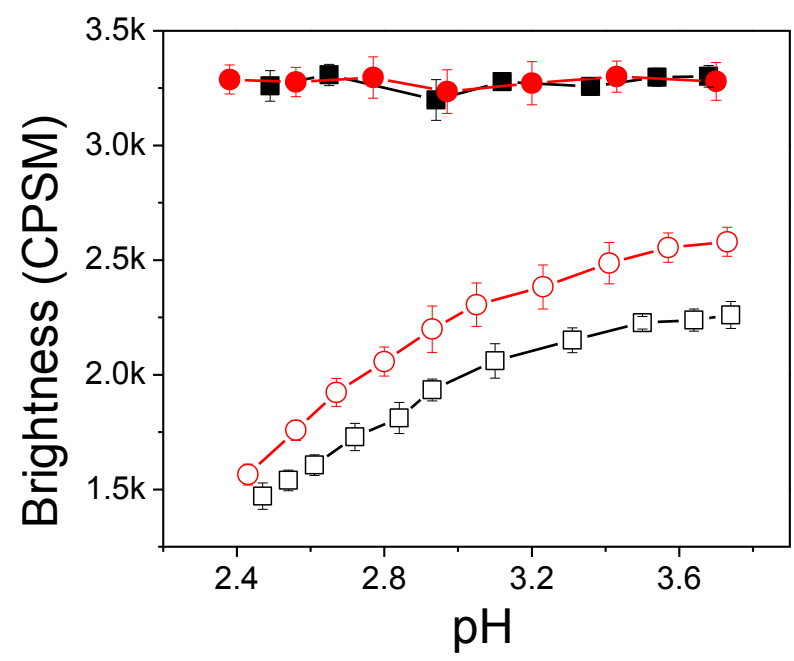

Figure S6. Measured fluorescence brightness, in the unit of photon counts per second per molecule (CPSM), of OG488 (open symbols) and Alexa488 (solid symbols) attached to the center (circles) and the periphery (squares) of P2VP stars in aqueous solution of varied $p H$. 
Statistics of PCH Data Analysis. We repeated the $\mathrm{PCH}$ experiment independently three times (shown in different color and cross-filled symbols) at the same environmental conditions with each coreand terminal-labeled P2VP star sample. For each experiment, the measured brightness data at each bulk $\mathrm{pH}$ were averaged as reported with error bars as shown in Figure S6 (A), which resulted from the fluctuation of fluorophore brightness in a certain period of time. Additionally, the local $\mathrm{pH}$ determined from the comparison to the $\mathrm{pH}-$ dependent brightness profile of free OG488 is shown in Figure $\mathrm{S} 7$ (B) with different symbol colors and crossed symbols representing each independent measurement. Clearly, the results obtained from different experiment sets are consistent with each other. More importantly, the errors are significantly smaller that the distinction between the P2VP stars with different fluorophore labeling locations.
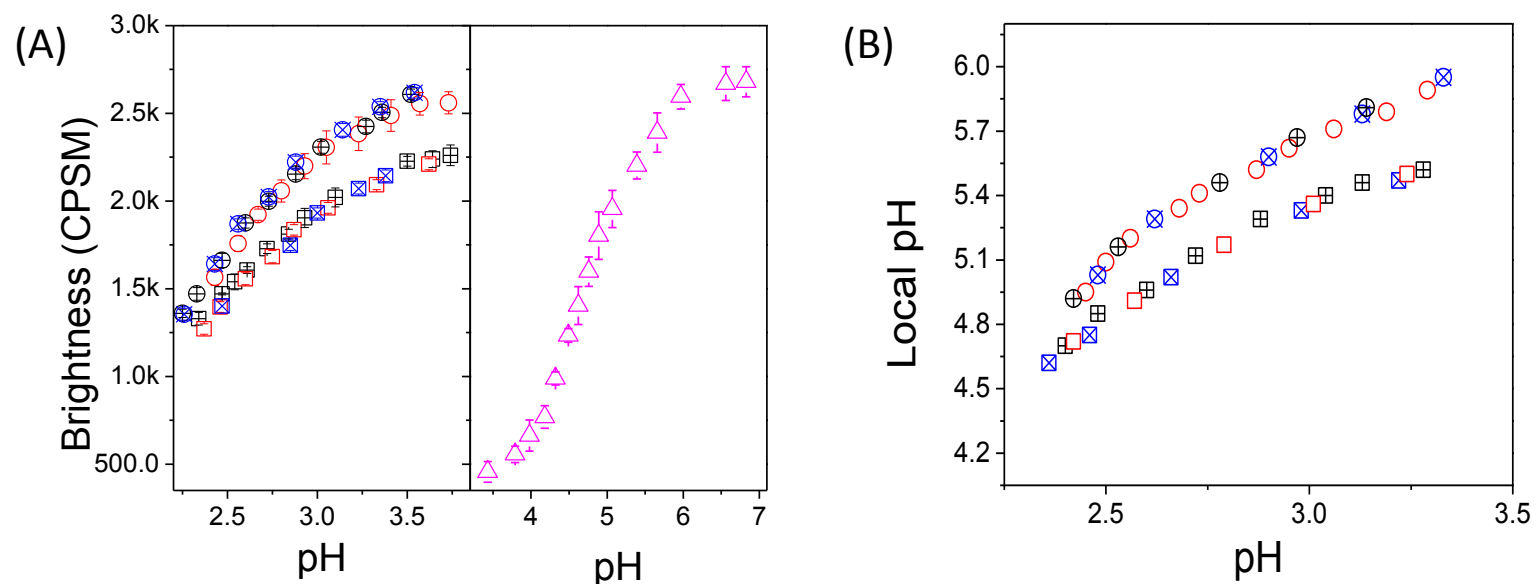

Figure S7. A) Measured fluorescence brightness (unit: CPSM), of free OG488 in P2VP-free $\mathrm{K}_{2} \mathrm{HPO}_{4} /$ citric acid buffer solution (triangles), OG488 attached to the center (circles) and the periphery (squares) of P2VP stars in aqueous solution of varied $\mathrm{pH}$. At each experimental condition, the experiment was repeated three times and results are shown in different colors and filled symbols. B) Local pH in the close vicinity to OG488 attached to the center (circles) and the periphery (squares) of P2VP stars. Results from repeated experiments are shown in different colors and filled symbols.

Results of Quantum Yields. As shown in Figure S8 (A), quantum yields of free and P2VP starattached OG488 were measured against solution $\mathrm{pH}$ to confirm the observation by PCH brightness measurement. An apparent increase in quantum yields is observed with increasing solution $\mathrm{pH}$ similar to the brightness change measured by PCH. Similar to the PCH analysis, the local pH probed by OG488 attached to the star center and the periphery of P2VP stars was obtained by comparing the quantum yields to the same value of free OG488 in the corresponding bulk $\mathrm{pH}$. The local $\mathrm{pH}$ obtained by $\mathrm{PCH}$ and quantum yield measurement agree well with each other as shown in Figure S8 (B), excluding the artifact 
introduced by the characterization methods and indicating the different electrostatic environment in the center from that in the periphery of P2VP stars.

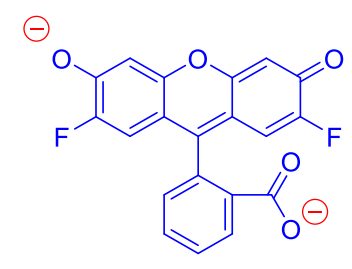

Dianion

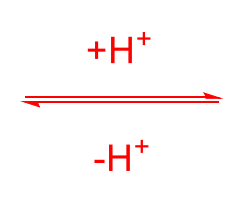

Monoanion
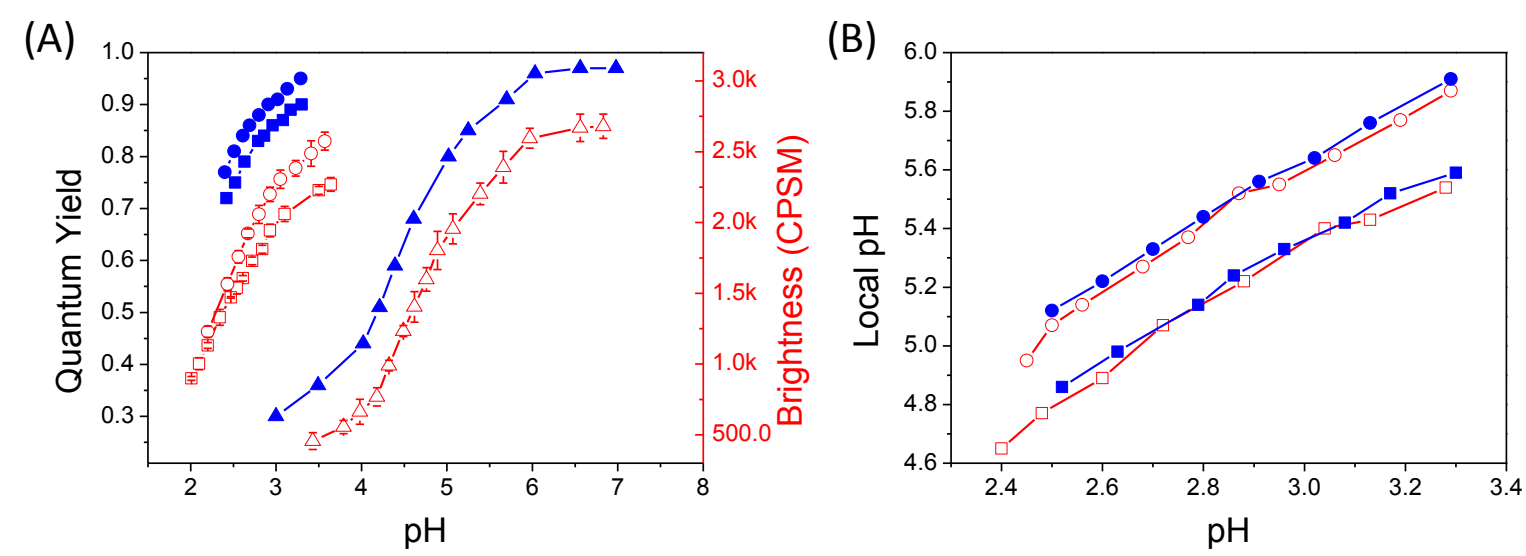

Figure S8. Schematic illustration the chemical equilibrium of the ionization of OG488 with proton concentration. A) Measured absolute quantum yields (show in left coordinate axis) of free OG488 (solid blue triangle), OG488 attached to the center (solid blue circles) and the periphery (solid blue squares) of P2VP stars against solution pH in comparison to the brightness results (shown in right coordinate axis) obtained by PCH show in red symbols of the same symbol shapes for different OG488 labeling. B) Local pH determined by quantum yield (blue) and PCH (red) measurements.

\section{References}

1. Ferguson, C. J.; Hughes, R. J.; Nguyen, D.; Pham, B. T. T.; Gilbert, R. G.; Serelis, A. K.; Such, C. H.; Hawkett, B. S., Macromolecules 2005, 38, 2191-2204.

2. $\quad$ Chen, M.; Moad, G.; Rizzardo, E., J. Polym. Sci., Part A: Polym. Chem. 2009, 47, 6704-6714.

3. Vink, H., Die Makromolekulare Chemie 1981, 182, 279-281.

4. $\quad$ Magde, D.; Elson, E.; Webb, W. W., Phys. Rev. Lett. 1972, 29, 705.

5. $\quad$ Rigler, R.; Mets, Ü.; Widengren, J.; Kask, P., Eur. Biophys. J. 1993, 22, 169-175.

6. Wang, S.; Zhu, Y., Soft Matter 2011, 7, 7410-7415.

7. Luo, S.; Jiang, X.; Zou, L.; Wang, F.; Yang, J.; Chen, Y.; Zhao, J., Macromolecules 2013, 46, 3132-3136.

8. $\quad$ Klein Wolterink, J.; Van Male, J.; Cohen Stuart, M.; Koopal, L.; Zhulina, E.; Borisov, O., Macromolecules 2002, 35, 9176-9190.

9. $\quad$ Borisov, O.; Zhulina, E., Eur. Phys. J. B 1998, 4, 205-217.

10. Zhulina, Y. B.; Borisov, O. V.; Birshtein, T. M., Polym. Sci. USSR 1988, 30, 780-788. 